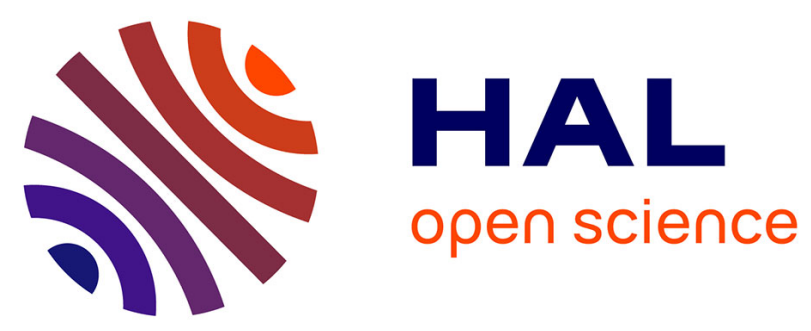

\title{
Microphone with planar nano-gauge detection: fluid-structure coupling including thermoviscous effects
}

Thierry Verdot, Emmanuel Redon, Kerem Ege, Jaroslaw Czarny, Cécile

Guianvarc'H, Jean-Louis Guyader

\section{- To cite this version:}

Thierry Verdot, Emmanuel Redon, Kerem Ege, Jaroslaw Czarny, Cécile Guianvarc'H, et al.. Microphone with planar nano-gauge detection: fluid-structure coupling including thermoviscous effects. Acta Acustica united with Acustica, 2016, 102 (3), pp.517-529. 10.3813/AAA.918969 . hal-01310567

\section{HAL Id: hal-01310567 \\ https://hal.science/hal-01310567}

Submitted on 12 May 2016

HAL is a multi-disciplinary open access archive for the deposit and dissemination of scientific research documents, whether they are published or not. The documents may come from teaching and research institutions in France or abroad, or from public or private research centers.
L'archive ouverte pluridisciplinaire HAL, est destinée au dépôt et à la diffusion de documents scientifiques de niveau recherche, publiés ou non, émanant des établissements d'enseignement et de recherche français ou étrangers, des laboratoires publics ou privés. 


\title{
Microphone with planar nano-gauge detection: fluid-structure coupling including thermoviscous effects
}

\author{
T. $\operatorname{Verdot}^{1 *}$, E. Redon ${ }^{1,2}$, K. Ege ${ }^{1}$, J. Czarny ${ }^{1,3}$, C. Guianvarc' ${ }^{1 \dagger}{ }^{1}$, J.-L. Guyader ${ }^{1}$ \\ 1) Univ Lyon, INSA-Lyon, LVA EA677, F-69621, Villeurbanne, France. \\ emmanuel.redon@insa-lyon.fr \\ 2) Université de Bourgogne, ESIREM, 9 Av. Alain Savary, BP 47 870, F-21078 Dijon,France. \\ 3) Univ. Grenoble Alpes, F-38000 Grenoble. CEA, LETI, MINATEC Campus, F-38054 Grenoble, France.
}

\begin{abstract}
This article presents the modeling of a MEMS microphone with an original architecture formed of mechanical structures moving in the plane of the substrate. On the contrary of most microphones generally constituted of an oscillating membrane, some transducers developed by the CEA-LETI with M\&NEMS technology use micro beams moving in the plane of the silicon wafer under the effect of an acoustical wave. These micro-structures are connected to the substrate by flexible micro-hinge and strain silicon nano-gauge producing a variation in resistance by piezoresistive effect. After the description of the design and functioning of the microphone under study, the vibroacoustic model of the fluid-structure coupling is presented. Considering the dimensions of the MEMS transducer close to the thermal and viscous boundary layers thicknesses, this model has to include diffusion phenomena. The model is discretized using the finite element method and the weak formulation is implemented using COMSOL Multiphysics ${ }^{\circledR}$ software. The pressure sensitivity of the microphone is calculated and compared with an analytical lumped model to asses the numerical model. Pressure and velocity fileds are also computed. Solutions of simulations are interpreted by focusing on phenomena influencing the sensitivity of this novel sensor design. In particular, the influence of the geometry and the role of the different part of the transducer (back cavity, mechanical structures) are studied.
\end{abstract}

The archived file is not the final published version of the article:

"T. Verdot, E. Redon, K. Ege, J. Czarny, C. Guianvarc'h, J.-L. Guyader, Microphone with planar nano-gauge detection: fluid-structure coupling including thermoviscous effects", in Acta Acustica united with Acustica, Vol. 103, 2016, pp. 517-529, ( ) (2016) S. Hirzel Verlag/European Acoustics Association.

The definitive publisher-authenticated version is available online at:

http://www.ingentaconnect.com/contentone/dav/aaua/2016/00000102/00000003/art00014,

http//dx.doi.org/10.3813/AAA.918969 (DOI: 10.3813/AAA.918969).

Readers must contact the publisher for reprint or permission to use the material in any form.

\section{Introduction}

Acoustic transducers have been dominated for decades by condenser microphones. Condenser microphones, invented by E.C. Wente [1] in 1916 are improved by G.

\footnotetext{
Received 12 November 2016, accepted 8 April 2016

* Current adress: Univ. Grenoble Alpes, F-38000 Grenoble. CEA, LETI, MINATEC Campus, F-38054 Grenoble, France. thierry.verdot@cea.fr

† Current adress: Laboratoire Commun de Métrologie LNE-Cnam, 61 rue du Landy, 93210 La Plaine Saint Denis, France.

cecile.guianvarc_h@cnam.fr
}

Sessler and J. West in 1962 [2] with the electret microphone. Electret microphones are of simple design and have good acoustic properties. Commercially available since 1968, electret microphones represent 80 to $90 \%$ of the worldwide microphone market until 2007. In recent years the field of acoustic sensor technology, has known significant innovations due to the silicon technology used for MEMS (Micro Electro Mechanical Systems) and the use of new electroactive materials. The first commercial MEMS microphone was presented in 2002 by Knowles, nearly 20 years after the report of the first working condenser microphone based on silicon micromachining by G. Sessler and D. Hohm in 1983 [3]. 
MEMS microphones use silicon that provides exceptional mechanical characteristics [4] along with good electrical properties and mature fabrication technology [5]. Regardless of the transduction principle (capacitive, piezoresistive, piezoelectric, optical), all of the MEMS microphones reported in the state of the art literature are based on a membrane deflecting out of the plane of the base wafer. Numerous surface micromachined membranes have been reported, among them those made of metalized silicon nitride [6, 7], the combination of silicon nitride and polysilicon [8], polysilicon [7], [9] and bulk micromachined membranes reported by Y. Iguchi [10]. Most of the reported microphones and all of the commercially available MEMS use capacitive transduction [11].

Microphones miniaturization is of great interest for several fields, such as medical applications (audio implants), or consumer electronics (cell phones, computers) and MEMS technology have significantly reduced their size since the early the 2000s. However, as indicated in [11], difficulties arise to maintain performances for condenser microphones with miniaturization. In particular, the Signal to Noise Ration (SNR) tends to decrease with the chip area.

In this field, a novel architecture of microphone without membrane have been developed by CEA-LETI. This microphone uses beams moving in the plane of the substrate and inducing strain on piezoresistive Si nano-gauges (M\&NEMS technology). This concept seems promising to increase the miniaturization without significant decrease in sensitivity. The M\&NEMS concept using piezoresistive detection with $\mathrm{Si}$ nano-gauges has already been demonstrated on inertial sensors such as gyroscopes [12], and results obtained on these sensors confirm the miniaturization potential of this new principle.

The conception and optimization of such planar piezoresistive microphone requires a good understanding of its acoustic and vibroacoustic behavior. Due to the small dimensions of the microphone's slits $(1-100 \mu \mathrm{m})$, the device modeling should take into account: a) viscous and thermal effects in the boundary layers, b) their coupling to the beams vibroacoustic behavior.

Analytical solutions to the Kirchhoff equations in viscothermal fluids [13] presenting a good agreement with experimental results, but only for acoustic devices with simpler shapes [14] or higher dimensions [15]. The microsystem studied has a complex shape, small dimensions and strong fluid-structure coupling. It is therefore necessary to use numerical modeling that take into account all the physical phenomena occurring in the microphone.

Several numerical modelings that use boundary element methods and finite element methods (BEM/FEM) have been carried out [16, 17, 18, 19, 20, 21, 22, 23]. Those models describe acoustic fields and/or vibroacoustic membranes and plates behaviors that are coupled to viscous and thermal diffusive fields in the boundary layers.

The aim of the present paper is to provide accurate and adapted analytical and numerical (FEM) models able to gather all these effects in a consistent manner. We present here the full and detailed vibroacoustic model with analytical comparisons, extending previous contributions [24, 25], where only parts of modelling process were given.

The outline of the paper is the following. The principle of the planar nano-gauge detection microphone is presented in the next section. The vibroacoustical model including viscothermal effects of the microphone is presented in section 3 and a simplified vibroacoustic model for the mechanical structures is introduced. Section 4 is devoted to the numerical implementation of the model using the finite element method. Especially, we specify the method used for the coupling between the acoustic medium, mechanical structures and the back volume. $\mathrm{Nu}$ merical results are presented in section 5 . The vibroacoustic model is first used to show the influence of viscothermal effects of the pressure gradient between the inlet and the back cavity. Finally the sensitivity of the microphone is estimated, and the influence of some geometric parameters are studied. The results are compared with those obtained using an analytical model.

\section{Principle of the planar nano-gauge de- tection microphone}

Figure 11-(a) gives an overview of the prototype microphone studied in this paper. This sensor, of small size $1.5 \times 1.5 \times 0.6 \mathrm{~mm}^{3}$, is realized by a manufacturing method in clean room. A network containing air for acoustic propagation is created, embedding sensitive mechanical micro-structures with a nano-gauge and make the electrical connections.

The cross section of the MEMS (Figure 1 1 (b) ) shows the stack of layers and the network of air circulation made in the micro-system. Vents are etched in the cover (Silicon wafer) and in the SOI (Silicon On Insulator) wafer in order to reach outside. The openings of vents, are the inputs and outputs of the acoustic network. Inside the micro-system, vents communicate by means of flat slots (couplers) where the sensitive mechanical micro-structures are located.

The arrangement of micro-structures is shown in the sectional view in the plane of the wafer (Figure 2). These micro-structures are formed by four truss beams (Figure 2 . (a)) connected to the substrate by a flexible micro-hinge (Figure 2.(b)) that allows their oscillation in the plane $\left(\mathbf{e}_{1}, \mathbf{e}_{3}\right)$ of the device. Above the hinge, the beams are connected to the frame by a strain gauge of nanometric cross section (Figure 2.(c)). The rotation of a beam generates significant longitudinal stress in the gauge and produces a variation in resistance by piezoresistive effect. The association of strain gauges in a Wheatstone bridge is used to detect the variation of resistance due to a pressure difference $\Delta p$ on either side of the beams (see Figure 3 .

The operating principle of the microphone can be represented by the block diagram (Figure 3), that shows the behaviors and interactions of the various components of the microphone: 


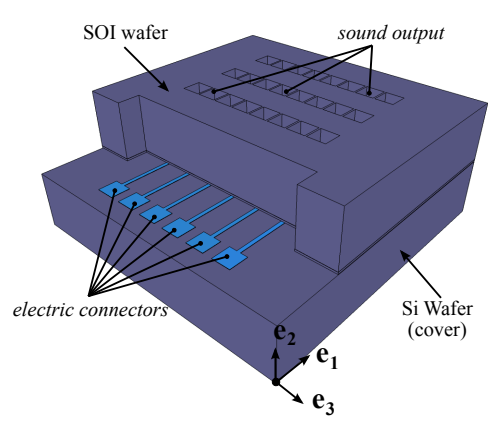

(a)

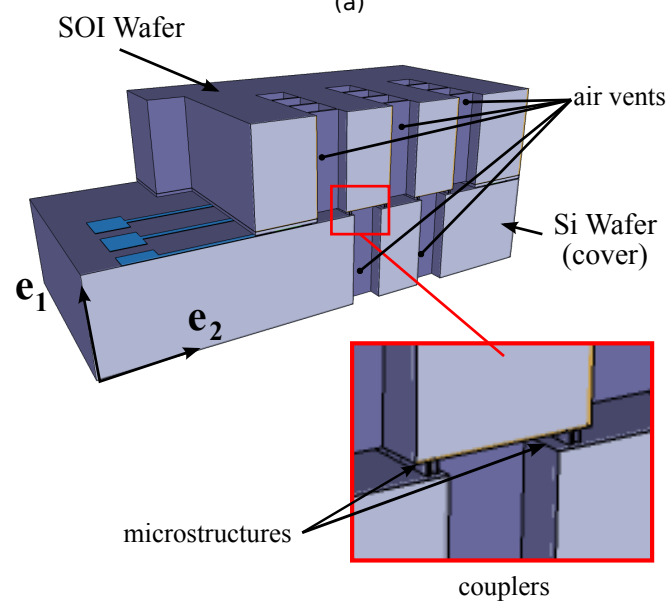

(b)

Figure 1. (a) Overview of the microphone MEMS, (b) sectional view in the thickness

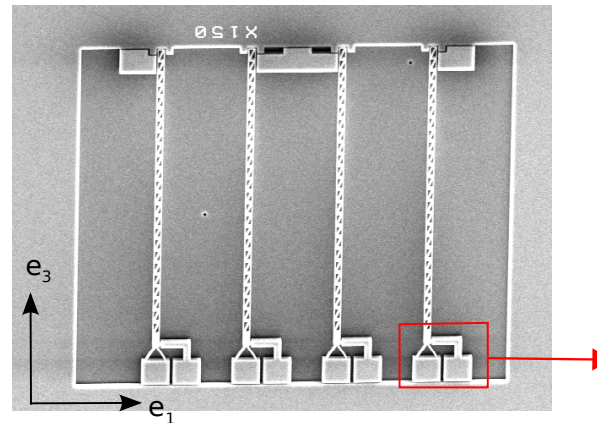

(a)
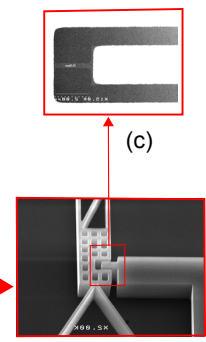

(b)

Figure 2. Section in the plane of the couplers (a) arrangement of sensitive structures, (b) micro-hinge of the beam, (c) nano-gauge

- the acoustic behavior of air in the micro-system allows the transmission of the incident pressure fluctuations and generates a torque $\left(\Gamma_{A}\right)$ on the beams,

- the mechanical behavior of the beams establishes the link between the torque imposed by the acoustic pressure and the rotation of the beams $(\theta)$,

- the electrical behavior of the piezo-resistive gauges, inserted in a Wheatstone bridge, links the measured voltages $(\Delta V)$ with the axial stresses in the gauges.

Thus, predicting the sensitivity of the micro-system consists in solving a coupled problem that is presented in details in the next section.

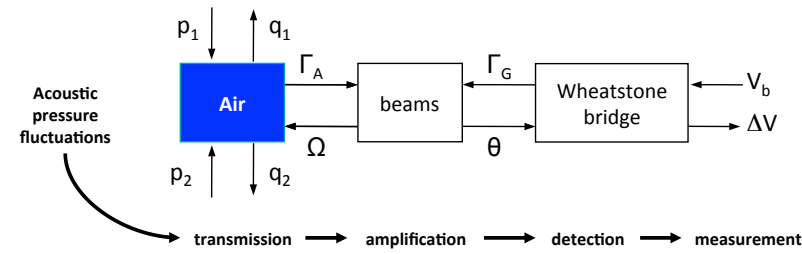

Figure 3. Schematic representation of the measurement device. $p_{1}$ and $p_{2}$ are the input and output pressure with $\Delta p=p_{1}-p_{2}$, $q_{1}$ and $q_{2}$ represent the resulting input and output volume velocity, $\Omega^{(i)}=\dot{\theta}^{(i)}$ is the rotational arms speeds of each beam $(i)$, $\Gamma_{A}^{(i)}$ are the torques applied on the beams due to $\Delta p$ the acoustic pressure difference, $\Gamma_{G}^{(i)}$ are the torques spring return due to the gauges, $V_{b}$ is the bias voltage and $\Delta V$ the voltage mesure at the Wheatstone bridge.

\section{Vibroacoustical model of the microphone}

Heat diffusion by conductivity and momentum diffusion by viscosity in the acoustic propagation medium are at the origin of the formation of the boundary layers near the walls of the domain. In these boundary layers, diffusion phenomena have a significant influence on the propagation of acoustic waves.

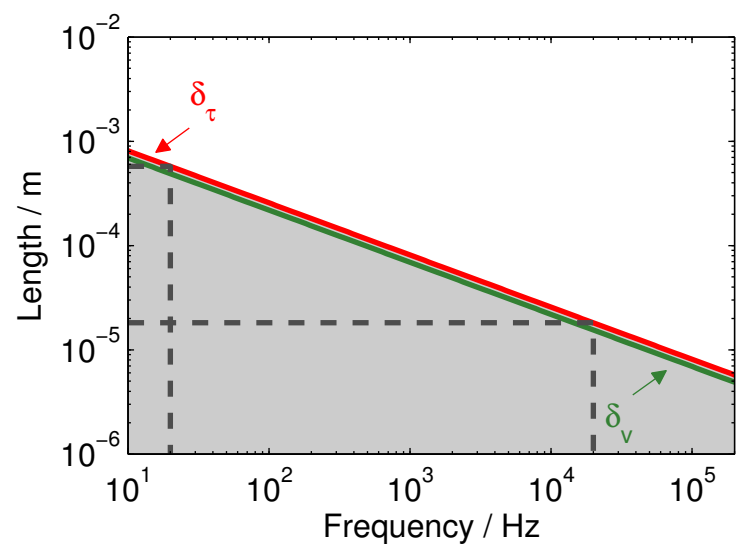

Figure 4. Thickness of thermal and viscous boundary layers in air $\left(P_{0}=1\right.$ bar $\left.T_{0}=20{ }^{\circ} \mathrm{C}\right)$. Audible range $(20 \mathrm{~Hz}-20 \mathrm{kHz})$ is indicated in dashed line - (from N. Joly [22])

Figure 4 reports the thickness and thermal characteristics of viscous boundary layers in air as a function of frequency $f$ (equation (1) from [22]) where $\omega=2 \pi f$ is the angular frequency of the acoustic wave:

$$
\delta_{v}(\omega)=\sqrt{\frac{2 \mu}{\rho_{0} \omega}} \quad \delta_{\tau}(\omega)=\sqrt{\frac{2 \kappa}{\rho_{0} c_{P} \omega}} .
$$

Under normal conditions of temperature and pressure ( 1 bar, $20{ }^{\circ} \mathrm{C}$ ), the density of air at rest $\rho_{0}$ is close to $1.2 \mathrm{~kg} / \mathrm{m}^{3}$, its specific heat capacity at constant pressure 
$c_{P}$ is close to $1 \mathrm{~kJ} / \mathrm{K} / \mathrm{kg}$, the coefficient of shear viscosity $\mu$ is close to $18 \mu \mathrm{Pa} \cdot \mathrm{s}$ and thermal conductivity $\kappa$ close to $25 \mathrm{~mW} / \mathrm{K} / \mathrm{m}$. For the audible frequency range $(20 \mathrm{~Hz}$ to $20 \mathrm{kHz}$ ), the thicknesses of the boundary layers vary from 600 to $20 \mu \mathrm{m}$.

Located in the vicinities of the walls, the dissipative effects are neglected in the acoustic conservative models used to study the propagation of acoustic waves in free field or in closed environments whose characteristic dimensions highly exceed the boundary layer. These models are based on an Helmholtz equation obtained by considering adiabatic pressure and reversible processes.

Micro-systems boundary layers occupy the major part of the fluid space. Hence, the study of acoustic behavior supposes to develop appropriate models to take into account the diffusion phenomena. For air, the thicknesses of the thermal and viscous boundary layers are of the same order of magnitude, these models necessarily take into account both diffusion processes and are called "thermoviscous".

\subsection{Viscothermal acoustics in the MEMS micro- phone}

The general formulation used for modeling the acoustic behavior in thermo-viscous propagation media is based on the Full Linearized Navier-Stokes equations (FLNS). The propagation of sound in a thermo-viscous fluid is based on the local balance of momentum (2), energy (3) and mass (4) linearized under the assumptions of small fluctuations around the equilibrium state.

These balance equations govern the velocity fluctuations $\mathbf{v}$, acoustic pressure $p$ and fluctuation of temperature $\tau$ of each particle (in the sense of continuum mechanics) of the domain. They also depend on the thermodynamic parameters describing the environmental conditions of the propagation medium (static pressure $P_{0}$, mean temperature $T_{0}$, the density $\rho_{0}$ of air at rest and $C_{P}$ the heat capacity with constant pressure). The air in the microphone being assumed at rest $\left(\mathbf{v}_{0}=0\right)$, the Full Linearized NavierStokes (FLNS) equations are written:

$$
\begin{aligned}
\rho_{0} \frac{\partial \mathbf{v}}{\partial t} & =\underline{\mathbf{f}}+\nabla \cdot \overline{\bar{\sigma}}=\underline{\mathbf{f}}-\nabla p+\nabla \cdot \overline{\bar{\sigma}}_{v} \\
\rho_{0} c_{P} \frac{\partial \tau}{\partial t}-\frac{\partial p}{\partial t} & =\underline{r}-\nabla \cdot \mathbf{q} \\
\frac{\partial \rho}{\partial t}+\rho_{0} \nabla \cdot \mathbf{v} & =0
\end{aligned}
$$

where $\underline{\mathbf{f}}$ is the exterior body force (per unit volume) and $\underline{r}$ the heat source.

In the balance of momentum (2), the flux term concerns the Cauchy stress tensor $\overline{\bar{\sigma}}=-p \overline{\overline{1}}+\overline{\bar{\sigma}}_{v}$ that takes into account the stresses generated by the pressure fluctuation and the viscous effects described by the stress tensor $\overline{\bar{\sigma}}_{v}$. In the thermodynamic balance 3 , the incoming heat flux q represents the heat flow established between the air particles by thermal conduction. In the mass balance (4), the divergence of the velocity vector $\nabla \cdot \mathbf{v}$ is the rate of volume expansion.

The viscous and thermal diffusion occur respectively through the divergence of the viscous stress tensor $\overline{\bar{\sigma}}_{v}$ and incoming heat flux vector $\mathbf{q}$. The air is a fluid assumed to be Newtonian with $\mu$ and $\mu$ the shear and secondary viscosity coefficient respectively. The expression of the viscous stress tensor is given by (5). The flow established between the gas particles by heat conduction is given by Fourier's law (6) where $\kappa$ denotes the thermal conductivity of air:

$$
\begin{aligned}
\overline{\bar{\sigma}}_{v} & =\mu^{\prime}(\nabla \cdot \mathbf{v}) \overline{\overline{\mathbb{1}}}+\mu\left(\nabla \mathbf{v}+\nabla \mathbf{v}^{T}\right), \\
\mathbf{q} & =-\kappa \nabla \tau .
\end{aligned}
$$

The density of air is dependent on a state variable related to the pressure and temperature by a balance relation. Assuming that the air behaves as an ideal gas, the average magnitudes verify the state relationship (7) where $r=R / M_{a i r}$ is the specific constant of air:

$$
P_{0}-\rho_{0} r T_{0}=0 \text {. }
$$

This balance relation is assumed to be satisfied for small fluctuations around the initial equilibrium state. It is used in the mass balance equation (4) to express the density fluctuation in terms of temperature and pressure:

$$
\frac{1}{\rho_{0}} \frac{\partial \rho}{\partial t}=\frac{1}{P_{0}} \frac{\partial p}{\partial t}-\frac{1}{T_{0}} \frac{\partial \tau}{\partial t}=-\nabla \cdot \mathbf{v} .
$$

\subsection{Simplified vibroacoustic model for mechanical structures}

The FLNS formulation of the acoustic problem has many unknowns (particle velocity, acoustic pressure and temperature fluctuation). Using the finite element method for the discretization of the FLNS model will thus lead to a very large number of degrees of freedom ( 5 degrees of freedom per node). Indeed, taking into account the thermoviscous effects requires a very fine mesh near the walls, in the thermal and viscous boundary layers. Consequently, modeling the acoustic field and the 3D mechanical structures would be too expensive for solving the full coupled system. We therefore tried to reduce the size of the model to reduce it to an equivalent $2 \mathrm{D}$ problem.

To simplify the real micro-system (Figure 1 -a), the vents are assimilated to thin slits over the entire length of the device (Figure 5-a). The fluid acoustic field can then be reduced to a $2 \mathrm{D}$ model represented by a section in the plan $\left(\mathbf{e}_{1}, \mathbf{e}_{2}\right)$ (see Figure 5-b).

Sensitive structures are beams fixed at one extremity by a hinge (see Figure 2-b) where the gauges are located. The kinematics of the beams being inconsistent with a 2D model, a simplified mechanical model was defined by switching the rotation beams with translation beams, assuming a link between the center and the hinge with a 


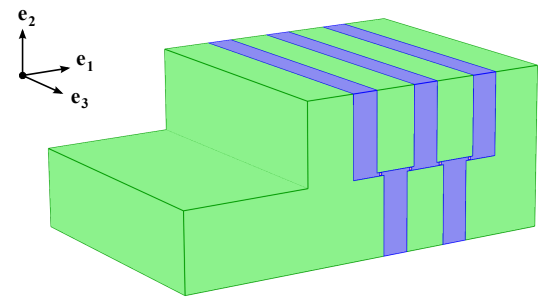

(a)

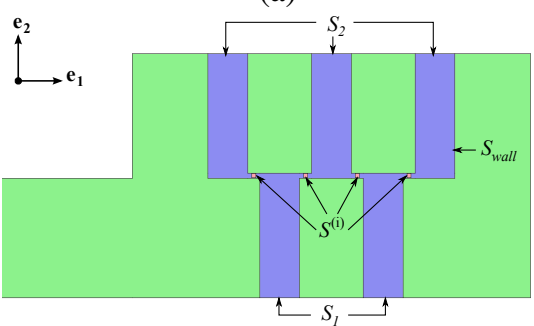

(b)

Figure 5. Simplified fluid acoustic domain: (a) 3D simplification, (b) equivalent $2 \mathrm{D}$ model.

massless arm (see Figure 6-a). The behavior of the mechanical system is similar to the real micro-system, and compatible with the 2D acoustic model, the beams then reduced to their cross section in translation in the plan $\left(\mathbf{e}_{1}, \mathbf{e}_{2}\right)$ along the $\mathbf{e}_{1}$ direction (see Figure 6 -b).

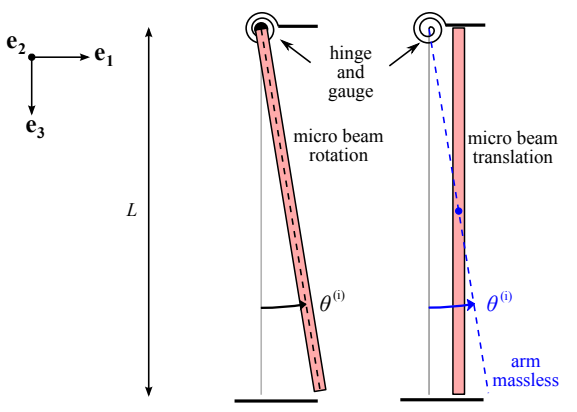

(a)

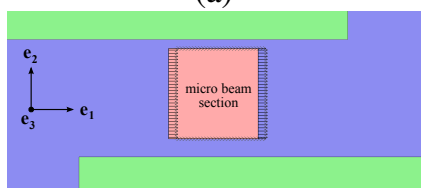

(b)

Figure 6. Simplified mechanical system: (a) modification of the kinematic, (b) mechanical system in the $2 \mathrm{D}$ model.

Massless arms are equipped with a flexible hinge and a gauge identical to those of the real micro-system. The total torque stiffness represented by a torsion spring is denoted: $C=C_{h}+d K_{g} d$, where $C_{h}$ is the torque stiffness of the hinge, $d$ is the lever arm (distance between the hinge and the gauge) and $K_{g}$ is the longitudinal stiffness of the gauge.

If $J$ denotes the moment of inertia of each beam $(i)$ of length $L$ evaluated at the rotational axis of the flexible hinge and $M$ their mass, the balance of moments on the beams and on the massless arm can be expressed respectively by:

$$
\begin{aligned}
& J \ddot{\theta}^{(i)}+C \theta^{(i)}=\frac{L}{2} S \Delta p \\
& \left(\frac{L}{2}\right) M\left(\frac{L}{2}\right) \ddot{\theta}^{(i)}+C \theta^{(i)}=\frac{L}{2} S \Delta p .
\end{aligned}
$$

where $\Delta p$ is the pressure difference between the two faces of the beams. The equations of motion being similar for both models, it is assumed that the mass of the translation model is $M(L / 2)^{2} \equiv J$ in order to have a simplified model with the same mechanical resonance frequency.

A uniform pressure distribution on the mechanical diaphragm leads to the same torque on the hinge for both models. Reciprocally, movement of the mechanical structure produced an identical volume velocity in acoustic model.

\subsection{Integration of MEMS and back cavity}

The microphone can work only if a pressure difference exists between the micro-system acoustic ports for loading the beam and thus the nano gauge. To avoid a short circuit in acoustic network, input and output acoustic ports are disconnected by the MEMS encapsulation's. "Top port" and "bottom port" configurations, shown in figure 7, are possible.

In the bottom-port configuration (figure 7 -a), the incident waves arrive to the microphone's input through a simple hole in the Printed Circuit Board (PCB), the top slits being loaded by the volume of the package, called back cavity. In the top-port configuration (figure 7 -b), incident acoustic waves arrive to the microphone's input through the hole in the shell of the package, the bottom slits being loaded acoustically by a small cavity in the PCB.

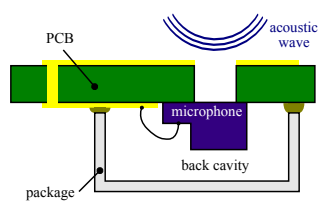

(a) Bottom port

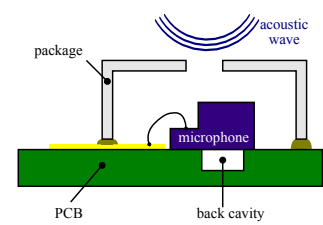

(b) Top port
Figure 7. Encapsulation configurations of the MEMS

The top port configuration is not considered in this study because the achievable back cavity into the PCB would be too small. Indeed, we consider here the bottom port configuration for which the back cavity is large compared to the size of the micro-system.

The characteristic dimensions of the cavity are small compared to the wavelengths considered, and large compared to the thickness of the boundary layer. To avoid costly and unnecessary finite elements discretization, the behavior of the back cavity is represented by a simplified model based on a lumped acoustic system [13]. The sound 
pressure $p_{c}$ is assumed uniform and heat transfers negligible $(\mathbf{q}=\mathbf{0})$ in the back cavity, the energy equation (3) then becomes:

$$
\rho_{0} c_{P} \frac{\partial \tau}{\partial t}=\frac{\partial p_{c}}{\partial t}
$$

The equation of mass conservation can be written in the back cavity:

$$
\frac{1}{\gamma P_{0}} \frac{\partial p_{c}}{\partial t}=-\nabla \cdot \mathbf{v}
$$

Finally, the pressure being uniform in the cavity, by integration, the acoustic pressure in the cavity $p_{c}$ is governed by the mass balance:

$$
C_{c} \frac{\partial p_{c}}{\partial t}=L \int_{S_{2}} \mathbf{v} \cdot \mathbf{n} \mathrm{d} \ell=Q_{c}
$$

where $C_{c}=V_{c} / \gamma P_{0}$ is the acoustic compliance of the back cavity of volume $V_{c}$, and where $Q_{c}$ denotes the volume velocity entering into the cavity through the rear surface $S_{2}$ (see figure 5-b). This equation will allow the coupling with the finite element model of the micro-system.

\section{Numerical implementation}

\subsection{Variational formulation}

Our models are based on a finite elements discretization of the variational formulation associated to the FLNS model (equations (2), (3), (4)). This variational formulation is obtained by the weighted residual method and leads to equation (14), which vanishes for the solution of the problem $(\mathbf{v}, \tau, p)$. It involves arbitrary weighting functions $\left(\mathbf{v}^{*}, \tau^{*}, p^{*}\right)$ defined over the entire domain and its boundaries:

$$
\begin{aligned}
& \mathcal{L}\left(\mathbf{v}^{*}, \tau^{*}, p^{*}, \mathbf{v}, \tau, p\right)=\cdots \\
& \int_{\mathcal{D}}\left[\mathbf{v}^{*} \cdot\left(-\rho_{0} \frac{\partial \mathbf{v}}{\partial t}\right)+\left(\nabla \cdot \mathbf{v}^{*}\right) p-\overline{\bar{\nabla}} \mathbf{v}^{*}: \overline{\bar{\sigma}}_{v}\right] \mathrm{d} V \cdots \\
& \quad+\int_{\mathcal{D}} \mathbf{v}^{*} \cdot \underline{\mathbf{f}} \mathrm{d} V+\int_{\partial \mathcal{D}} \mathbf{v}^{*} \cdot \overline{\bar{\sigma}} \mathbf{n} \mathrm{d} S \\
& +\int_{\mathcal{D}}\left[\frac{\tau^{*}}{T_{0}}\left(-\rho_{0} c_{P} \frac{\partial \tau}{\partial t}+\frac{\partial p}{\partial t}\right)+\frac{\nabla \tau^{*} \cdot \mathbf{q}}{T_{0}}\right] \mathrm{d} V \cdots \\
& \quad+\int_{\mathcal{D}} \frac{\tau^{*}}{T_{0}} \underline{r} \mathrm{~d} V+\int_{\partial \mathcal{D}} \frac{\tau^{*}}{T_{0}} \mathbf{q} \cdot(-\mathbf{n}) \mathrm{d} S \\
& +\int_{\mathcal{D}}\left[p^{*}\left(-\frac{1}{P_{0}} \frac{\partial p}{\partial t}+\frac{1}{T_{0}} \frac{\partial \tau}{\partial t}\right)-p^{*}(\nabla \cdot \mathbf{v})\right] \mathrm{d} V .
\end{aligned}
$$

The variational formulation (14) expressed in the time domain can be described as follow:

(i) the first three integrals in the right-hand side are associated with mechanical balance,

(ii) the following three integrals are associated with thermal balance, (iii) the last integral correspond to the mass balance.

These variational formulation (14) is used to calculate the time response to any excitation and investigate problems in transient regime. The harmonic regime also used for numerical results will be discussed in section 5

\subsection{Boundary conditions for acoustic cavity}

Output and input air vents $S_{1}$ and $S_{2}$ (Figure 5-(b)) are open boundaries, the acoustic velocity can evolve freely under the effect of pressure fluctuations $p_{1}$ and $p_{2}$ imposed by the external environment which may correspond to the inlet or back cavity (Figure 7). The temperature is also free to fluctuate assuming no heat transfer with the external environment. The boundary conditions of the input and output of the micro-system are:

$$
\begin{aligned}
& S_{1}\left\{\begin{array}{l}
\left(-p \overline{\overline{\mathbb{1}}}+\overline{\bar{\sigma}}_{v}\right) \mathbf{n}=-p_{1} \mathbf{n} \\
\mathbf{q} \cdot(-\mathbf{n})=0,
\end{array}\right. \\
& S_{2}\left\{\begin{array}{l}
\left(-p \overline{\overline{\mathbb{1}}}+\overline{\bar{\sigma}}_{v}\right) \mathbf{n}=-p_{2} \mathbf{n} \\
\mathbf{q} \cdot(-\mathbf{n})=0,
\end{array}\right.
\end{aligned}
$$

where $\mathbf{n}$ is the outgoing unit normal vector of the acoustic field.

The walls of the substrate, denoted $S_{\text {wall }}$, are assumed fixed, rigid, and with constant temperature. The adhesion condition to the wall imposes no acoustic velocity and temperature fluctuation:

$$
S_{\text {wall }}\left\{\begin{array}{l}
v_{1}=v_{2}=0 \\
\tau=0
\end{array}\right.
$$

Taking into account the boundary conditions in the variational formulation (14) for the simplified 2D problem ( $\mathrm{d} V$ becomes $\mathrm{d} S$, and $\mathrm{d} S$ becomes $\mathrm{d} \ell$ ), leads to the variational formulation for the acoustic problem $\mathcal{L}_{a c}$ :

$$
\begin{aligned}
& \mathcal{L}_{a c}\left(\mathbf{v}^{*}, \tau^{*}, p^{*}, \mathbf{v}, \tau, p\right)=\cdots \\
& L \int_{\mathcal{D}}\left[\mathbf{v}^{*} \cdot\left(-\rho_{0} \frac{\partial \mathbf{v}}{\partial t}\right)+\left(\nabla \cdot \mathbf{v}^{*}\right) p-\nabla \overline{\overline{\mathbf{v}}}^{*}: \overline{\bar{\sigma}}_{v}\right] \mathrm{d} S \\
& +L \int_{\mathcal{D}}\left[\frac{\tau^{*}}{T_{0}}\left(-\rho_{0} c_{P} \frac{\partial \tau}{\partial t}+\frac{\partial p}{\partial t}\right)-\kappa \frac{\nabla \tau^{*} \cdot \nabla \tau}{T_{0}}\right] \mathrm{d} S \\
& +L \int_{\mathcal{D}}\left[p^{*}\left(-\frac{1}{P_{0}} \frac{\partial p}{\partial t}+\frac{1}{T_{0}} \frac{\partial \tau}{\partial t}\right)-p^{*}(\nabla \cdot \mathbf{v})\right] \mathrm{d} S \\
& +L \int_{S_{1}} \mathbf{v}^{*} \cdot\left\{-p_{1} \mathbf{n}\right\} \mathrm{d} \ell+L \int_{S_{2}} \mathbf{v}^{*} \cdot\left\{-p_{2} \mathbf{n}\right\} \mathrm{d} \ell,
\end{aligned}
$$

where $L$ is the thickness of the micro-system, according to $\mathbf{e}_{3}$ (see Figure 5 - a).

\subsection{Acoustic model coupling with the beams}

The fluid-structure coupling is achieved by the method of Lagrangian multipliers, imposing the continuity of velocity between the air and the beams to the fluid-structure interface $S^{(i)}$. 
The balance equations of mechanical structures 90 are written in a state system form, retaining rotational arms speeds $\Omega^{(i)}=\dot{\theta}^{(i)}$ as independent state variables. The structures are free, because the coupling with the acoustic model is not yet realized:

$$
\left[\begin{array}{ll}
J & 0 \\
0 & C
\end{array}\right]\left[\begin{array}{c}
\dot{\Omega}^{(i)} \\
\dot{\theta}^{(i)}
\end{array}\right]=\left[\begin{array}{cc}
0 & -C \\
C & 0
\end{array}\right]\left[\begin{array}{c}
\Omega^{(i)} \\
\theta^{(i)}
\end{array}\right]
$$

As for the substrate, the continuity of the velocity and the isothermal condition must be verified on the walls of the mobile beams $S^{(i)}$. According to the simplified model described in section 3.2, the boundary conditions for a beam $S^{(i)}$, moving in translation in the plane $\left(\mathbf{e}_{1}, \mathbf{e}_{3}\right)$ and linked at $L / 2$ with a massless arm (see Figure 6-(a)), are given by:

$$
S^{(i)}\left\{\begin{array}{l}
v_{1}=(L / 2) \Omega^{(i)} \\
v_{2}=0 \\
\tau=0 .
\end{array}\right.
$$

The fluid-structure coupling is introduced in the model with the adhesion condition, which reflects the continuity of the velocity of the beam and the air velocity:

$$
\frac{L}{2} \Omega^{(i)}-v_{1}=0 .
$$

These conditions are introduced into the variational formulation by adding a new unknown, the Lagrangian $\lambda$, on the mobile beams $S^{(i)}$. The variational formulation corresponding to the vibroacoustic problem for the MEMS is then written:

$$
\begin{aligned}
& \mathcal{L}_{\text {mems }}\left(\mathbf{v}^{*}, \tau^{*}, p^{*}, \Omega^{(i)^{*}}, \theta^{(i)^{*}}, \lambda^{*}, \mathbf{v}, \tau, p, \Omega^{(i)}, \theta^{(i)}, \lambda\right)= \\
& \mathcal{L}_{a c}\left(\mathbf{v}^{*}, \tau^{*}, p^{*}, \mathbf{v}, \tau, p\right) \cdots \\
& \quad+\sum_{i=1}^{4} \Omega^{(i)^{*}}\left(-J \cdot \frac{\partial \Omega^{(i)}}{\partial t}-C \theta^{(i)}\right) \ldots \\
& \quad+\sum_{i=1}^{4} \theta^{(i)^{*}}\left(-C \frac{\partial \theta^{(i)}}{\partial t}+C \Omega^{(i)}\right) \ldots \\
& \quad+\sum_{i=1}^{4} L \int_{S^{(i)}} \lambda^{*}\left\{\frac{L}{2} \Omega^{(i)}-v_{1}\right\} \mathrm{d} \ell \cdots \\
& \quad+\sum_{i=1}^{4} \Omega^{(i)^{*}} \frac{L}{2}\left(L \int_{S^{(i)}} \lambda \mathrm{d} \ell\right) \ldots \\
& \quad+\sum_{i=1}^{4} L \int_{S^{(i)}} v^{*}(-\lambda) \mathrm{d} \ell .
\end{aligned}
$$

The expression (21) of the vibroacoustic problem in the MEMS includes:

(i) the functional $\mathcal{L}_{a c}$ established for the acoustic problem (17) corresponding to the loading pressure on the acoustic input air vents $\left(S_{2}\right)$, (ii) the second and third terms in the right-hand side of 21) corresponding to the residues formed from the mechanical problem $(18$,

(iii) the fourth corresponding to the residues formed from adhesion conditions 20,

(iv) the last two terms corresponding respectively to the torques applied on the mechanical structures $\Gamma_{A}^{(i)}=\frac{L}{2}\left(L \int_{S^{(i)}} \lambda \mathrm{d} \ell\right)$, and the reaction forces which balance the stresses exerted by the air to the walls $-\lambda=\mathbf{e}_{1} \cdot\left(-p \overline{\overline{\mathbb{1}}}+\overline{\bar{\sigma}}_{v}\right) \mathbf{n}$.

\subsection{Acoustic model coupling with the back cavity}

The back cavity has an important role in the functioning of the micro-system, it behaves like an acoustic load to be taken into account in the model. This acoustic load depends on the evolution of the acoustic pressure in the back cavity, whose behavior is described by the equation 13 . The volume velocity of air entering in the back cavity $\left(Q_{c}\right)$ is evaluated from the normal acoustic velocity $(\mathbf{v} \cdot \mathbf{n})$ in the finite element model. Equation (13) can thus be implemented to provide pressure fluctuation in the back cavity:

$$
Q_{c}=C_{c} \frac{\partial p_{c}}{\partial t}=L \int_{S_{1}} \mathbf{v} \cdot \mathbf{n} \mathrm{d} \ell .
$$

The pressure fluctuation in the back cavity is now a global variable of the model that can be applied as a load on the acoustic port of the SOI by the boundary condition $(15)$ prescribing $p_{2}=p_{c}$.

\subsection{Finite Element Model implementation}

The variational formulations associated with the simplified acoustic model (17) or to the entire vibroacoustic model (21) are implemented in COMSOL MULTIPHYSICS 3.4 ® using PDE Modes module with weak form formulation. This specific model, including thermoviscous effects, does not exist in this version.

In accordance with the simplified 2D model described above, the geometry and mesh of the acoustic field are made. The element size, as specified in Figure 8 , is set at $4 \mu \mathrm{m}$ on the walls of the substrate in order to obtain enough elements in the boundary layers (at least 3 in the boundary layers, whose thickness is $15 \mu \mathrm{m}$ at $20 \mathrm{kHz}$ ). A very fine mesh is used in the couplers where the viscous and thermal effects are dominant. The order of the polynomial interpolation functions respects the Inf-Sup condition mentioned by Kampinga [23]: quadratic for velocity and temperature fields and linear to the pressure field.

Table I contains the values of the physical parameters of the acoustic model. The behavior of the air at rest in normal conditions of temperature $\left(20^{\circ} \mathrm{C}\right)$ and pressure $(1$ bar) is similar to that of a diatomic ideal gas. 


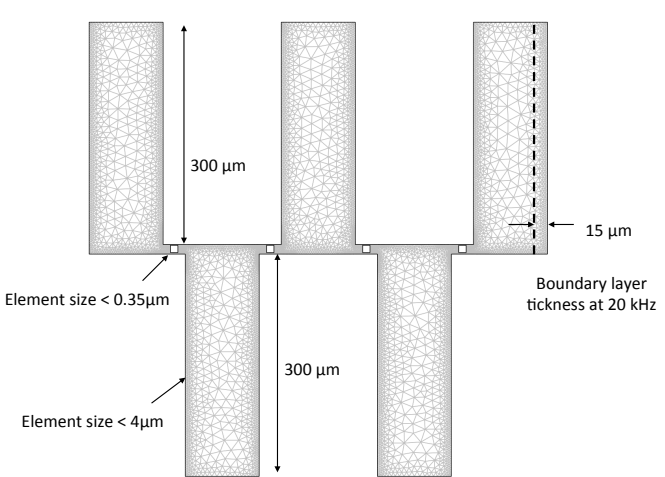

(a)

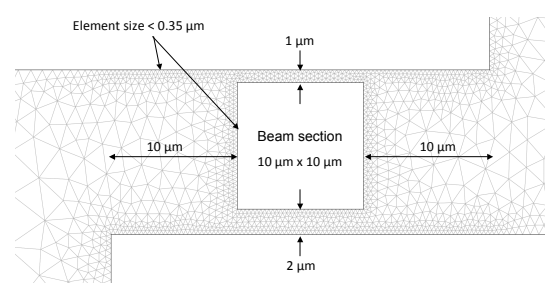

(b)

Figure 8. Mesh used for the discretization: (a) Mesh of the acoustic domain, (b) Detail of the mesh in a coupler

\begin{tabular}{|c|c|c|}
\hline \multicolumn{3}{|c|}{ Physical parameters of the air at rest } \\
\hline $\begin{array}{l}\text { Static pressure } \\
\text { Temperature } \\
\text { Molar mass of air } \\
\text { Specific constant air } \\
\text { Density } \\
\text { Heat capacity (P cte) } \\
\text { Heat capacity (V cte) } \\
\text { Heat capacity ratio }\end{array}$ & $\begin{aligned} P_{0} & = \\
T_{0} & = \\
M_{m o l} & = \\
r & = \\
\rho_{0} & = \\
c_{P} & = \\
c_{V} & = \\
\gamma & =\end{aligned}$ & $\begin{array}{l}101325 \mathrm{~Pa} \\
293.15 \mathrm{~K}\left(20^{\circ} \mathrm{C}\right) \\
28.96 \mathrm{~g} / \mathrm{mol} \\
287.1 \mathrm{~J} / \mathrm{K} / \mathrm{kg} \\
1.2 \mathrm{~kg} / \mathrm{m}^{3} \\
1004.9 \mathrm{~J} / \mathrm{K} / \mathrm{kg} \\
717.7 \mathrm{~J} / \mathrm{K} / \mathrm{kg} \\
1.4\end{array}$ \\
\hline \multicolumn{3}{|l|}{ Diffusion coefficients } \\
\hline $\begin{array}{l}\text { Heat Conductivity } \\
\text { Shear Viscosity } \\
\text { Bulk Viscosity } \\
\text { Second viscosity }\end{array}$ & $\begin{aligned} \kappa & = \\
\mu & = \\
\eta & = \\
\mu_{B} & =\end{aligned}$ & $\begin{array}{l}25 \mathrm{~mW} / \mathrm{K} / \mathrm{m} \\
18.10^{-6} \mathrm{~Pa} \cdot \mathrm{s} \\
0.60 \mu \\
\eta-2 \mu / 3\end{array}$ \\
\hline
\end{tabular}

Table I. Acoustic model parameters

\section{Numerical results}

A basic question is to identify the influence of viscothermal effects on the microphone performance. The detection principle is based on the solicitation of the piezoresistive gauges resulting from the movement of the beams. Then, it is clear that the operation of the microphone is conditioned by the existence of a pressure difference between the inlet and the back cavity to ensure the vibration of the beams.

Vibroacoustic microphone models established previously are now used to show the influence of viscothermal effects on the pressure difference in the MEMS and to determine the microphone sensitivity based on design parameters of the MEMS.
The finite elements discretization of the temporal variational formulation (21) allows us to calculate the time response of the coupled vibroacoustic problem in order to study the transient regime. For the sensitivity study, the harmonic formulation is required: it is easily obtained from 21) by replacing the time derivative by $j \omega$. Both approaches are used in the next sections.

\subsection{Evolution of the pressure in the back cavity}

Figure 9 presents the transient evolution of the pressure in the back cavity in response to a unit step input pressure of $1 \mathrm{~Pa}$ on the microphone input at boundary $S_{1}$. It shows that the characteristic time $\tau$ needed for the establishment of the pressure equilibrium in the back cavity is about $50 \mathrm{~ms}$. This time depends of the viscous resistance of the micro-system and the back cavity volume which modify the equivalent acoustic compliance.

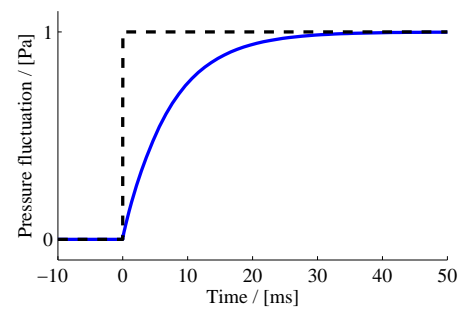

Figure 9. Evolution of the pressure in the back cavity of the microphone in response to a unit step pressure input of $1 \mathrm{~Pa}$.

This characteristic time $\tau$ has an important role in the working of the microphone, it should not be too short to ensure the existence of a pressure difference between the input and the output of the MEMS.

After examining the transient evolution of the acoustic pressure in the cavity, we now focus on the time evolution of the pressure in the cavity in response to an harmonic pressure fluctuation of $1 \mathrm{~Pa}$ of magnitude, imposed on the microphone input for different frequencies.

Figure 10-(b) corresponds to an excitation at $f=f_{1}=$ $22.48 \mathrm{~Hz}$. This cut-off frequency corresponds to the characteristic time shown in the previous figure $\tau=1 / f_{1}$. At this frequency, the pressure fluctuation in the back cavity and the pressure difference generated between the two acoustic ports have similar amplitudes $(1 / \sqrt{2})$ with $\pm 45^{\circ}$ phase difference with the imposed pressure (see red and blue curves).

The time responses to two other imposed fluctuations of frequency $f=2 \mathrm{~Hz}$ and $f=200 \mathrm{~Hz}$ are presented in figures (a) and (c) respectively. When a slow pressure fluctuation is imposed (500 $\mathrm{ms}$ of period) the pressure in the back cavity follows the input excitation (see blue and black curves in figure (a)). At this frequency $(f=2 \mathrm{~Hz})$ the pressures are balanced: the difference in pressure generated between the two acoustic ports has a small amplitude with about $90^{\circ}$ phase difference. 
When a rapid fluctuation is imposed $(5 \mathrm{~ms}$ of period, corresponding to $f=200 \mathrm{~Hz}$ ), there is no time for pressure balance and the difference in generated pressure is close to the acoustic pressure fluctuation imposed at the input (same amplitude and in phase, see figure-(c)).

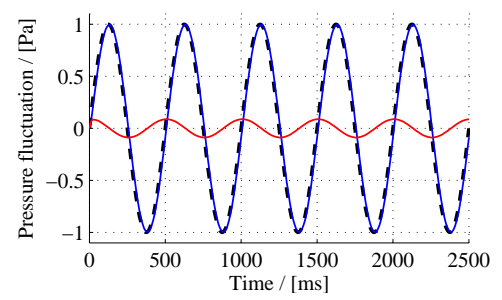

(a) $f=2 \mathrm{~Hz}$

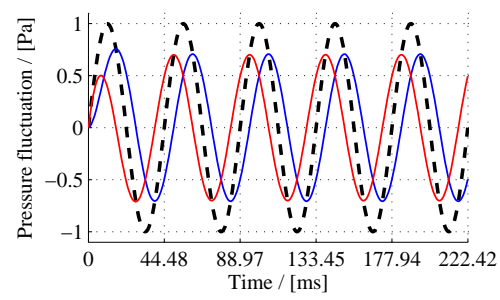

(b) $f=22.48 \mathrm{~Hz}$

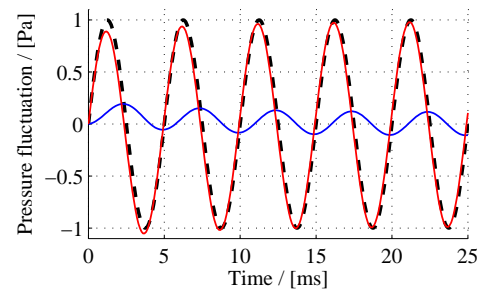

(c) $f=200 \mathrm{~Hz}$

Figure 10. Time response to an imposed harmonic pressure fluctuation $p_{1}(t)=1[P a] \sin (2 \pi f t)$ (dashed black curves) for three different frequencies. Pressure fluctuation in the back cavity (blue curve) and pressure difference between the acoustic ports (red curve).

In order to observe the evolution of the pressure difference on the audible frequency range, the amplitude and phase of the responses (Bode plots) obtained from calculations in harmonic regime are presented in figure 11 where $\tilde{p}_{c}$ is the complex pressure in the back cavity and $\tilde{p}_{1}$ is the complex input pressure. The cut-off frequency $f_{1}$ is indicated with the first vertical dashed line. Above this frequency, the Bode plot for the difference in pressure $\left(\tilde{p}_{1}-\tilde{p}_{c}\right)$ exhibits an exceptionally flat response. The amplitude of the pressure fluctuation in the back cavity decreases significantly and the pressure difference obtained follows the imposed pressure.

Figure 12 presents the pressure field and the velocity profile (real parts) in the coupler in harmonic regime at $1 \mathrm{kHz}$ and $13 \mathrm{kHz}$. It shows how the viscous effects produce the pressure difference on both sides of the beams. The velocity profiles obtained confirm that the model used is operational for the fluid-structure coupling.

Two different behaviors are revealed:
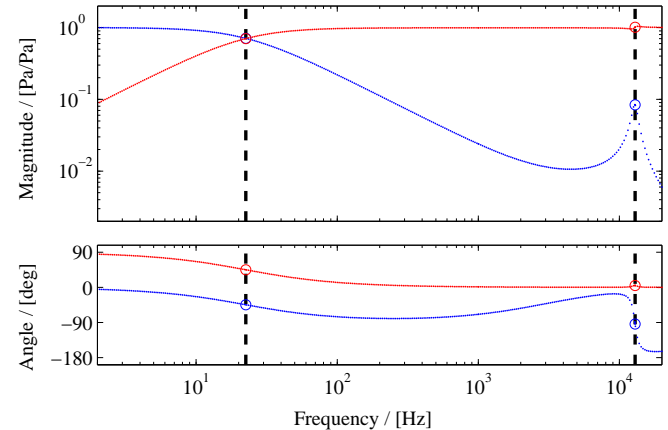

Figure 11. Frequency responses (Bode plots) in permanent harmonic regime $\tilde{p}_{c} / \tilde{p}_{1}$ (blue curve) and $\left(\tilde{p}_{1}-\tilde{p}_{c}\right) / \tilde{p}_{1}$ (red curve).

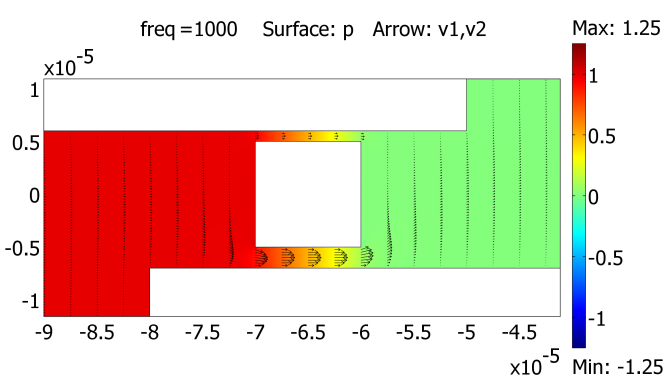

(a) $f=1 \mathrm{kHz}$

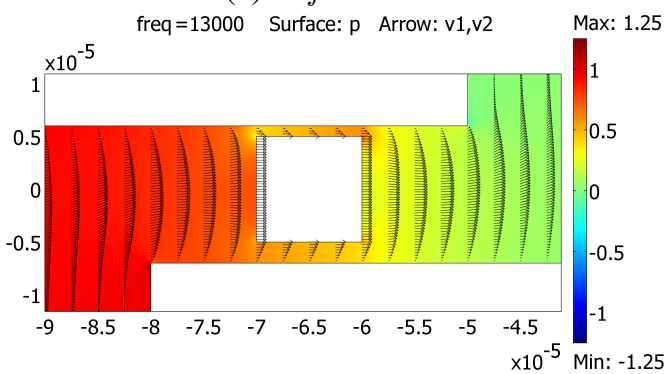

(b) $f=13 \mathrm{kHz}$

Figure 12. Pressure field (in color) and velocity profiles (arrows) in the coupler for a square section beam. Response to a unit harmonic pressure at $1 \mathrm{kHz}$ and $13 \mathrm{kHz}$ imposed on $S_{1}$ (Figure 5-b).

(a) At $1 \mathrm{kHz}$, the velocity of the beam is low and the air goes around the mechanical structures to establish a flow towards the back cavity. The velocity profile in the interstice gaps is governed by the pressure difference that exists between the acoustic ports of the microphone. This flow profile is parabolic, close to a Poiseuille flow.

(b) At $13 \mathrm{kHz}$, in the vicinity of the resonant frequency of the mechanical structures the velocity profile in the coupler is governed by the velocity of the structures. In the interstice gaps the flow profile is linear, close to a $\mathrm{Cou}$ ette flow. The movement of thestructures establishes a wall flow towards the back cavity resulting in a peak in the transfer amplitude $\tilde{p}_{c a v} / \tilde{p}_{i n}$ (see figure 11 .

Hence for simulated thicknesses of gaps (around the micrometer) and above the cut-off frequency $f_{1}$, the viscous effects in the coupler generate a differential in pressure proportional to the input pressure fluctuation. 


\subsection{Pressure sensitivity}

The previous sections introduced the elements of the considered microphone: the acoustic system of the MEMS dice, the chip-scale package, the mechanical structure and the electrical system constituted by the four piezoresistive nano-gauges arranged into a full Wheatstone bridge architecture.

According to the transduction chain presented on figure 3. these elements have to be assembled in order to estimate the microphone transfer function and sensitivity.

\subsubsection{Approximate form of sensitivity}

The Vibroacoustic system of the MEMS dice has been studied with the FEM model in the previous section taking into account the influence of the back-volume. Recall that the mechanical system (the micro-beams) is coupled to the acoustic system with the pressure gradient $\Delta p$ generated across the micro-beams. The movement of the micro-beams introduces a flow rate in the acoustic system. Finally, the mechanical system is coupled to the electrical system (Wheatstone bridge) by the longitudinal stress $\sigma_{g}$ generated inside the nano-gauges.

To estimate the sensitivity of the microphone, it is necessary to estimate each transfer transduction chain (see figure 3), starting with the electrical transfer:

- The relative variation of nano-gauge resistance $\Delta R / R$ is proportional to the longitudinal stress $\sigma_{g}$ applied to the nano-gauge due to the displacement of the beam:

$$
\Delta R / R=\pi_{p z r} \sigma_{g}
$$

where $\pi_{p z r}$ is the piezoresistive coefficient.

Accurate measurements of $\Delta R / R$ can be carried out with a Wheatstone bridge where output voltage is proportional to the variation resistance:

$$
\Delta V=V_{b}\left(\frac{\Delta R}{R}\right)=V_{b} \pi_{p z r} \sigma_{g}
$$

and the electrical transfer function becomes:

$$
T_{E}=\frac{\Delta V}{\sigma_{g}}=V_{b} \pi_{p z r}
$$

- The mechanical transmissibility of the microphone is expressed by the value of longitudinal stress in a nanogauge $\sigma_{g}$ generated by the rotation of the beam under a pressure gradient $\Delta p$. The quasi-static value of longitudinal stress is given by:

$$
\sigma_{g}=\frac{K_{g} u}{S_{g}}=\frac{K_{g} \theta d}{S_{g}}
$$

where $K_{g}$ denotes the longitudinal stiffness of a nanogauge with $S_{g}$ section, $u=\theta d$ is the displacement resulting of a rotation $\theta$ of the beam, $d$ being the distance between the hinge and the nano-gauge.
The rotation $\theta$ of the beam can also be deduced from the balance of the moments of the beam as a function of the pressure gradient:

$$
\theta=\frac{L}{2} \frac{S_{d}^{*} \Delta p}{C_{h}+d K_{g} d}
$$

where $S_{d}^{*}$ denotes the lateral surface of the beam $S_{d}$ that is modified due to the viscous effects, $C=C_{h}+d K_{g} d$ the total torque stiffness, $L / 2$ is the center of the beam.

In order to introduce the inertial effects we consider fluctuation of pressure at angular frequency $\omega$ and mechanical resonance of a beam at $\omega_{0}$, mechanical transfer function $T_{M}(\omega)=\sigma_{g} / \Delta p$ can be written:

$$
T_{M}=\left[\frac{S_{d}^{*}}{S_{g}}\right]\left[\frac{L / 2}{d}\right] \frac{d K_{g} d}{C_{h}+d K_{g} d}\left[\frac{1}{1-\left(\omega / \omega_{0}\right)^{2}}\right]
$$

Notice that structural damping and viscous damping are neglected here. However, viscous damping will be taken into account in the vibroacoustic coupled problem for the estimation of pressure differential $\Delta p$.

- The total sensitivity of a microphone is the combination of its acoustical, mechanical and electrical transfer functions. For the purpose of sensitivity analysis a general equation that describes the sensitivity can then be written from equations 25, 26, and (28) as follow:

$$
\begin{aligned}
& S(\omega)=\frac{\Delta V}{p_{1}}=T_{E} \cdot T_{M} \cdot T_{A} \\
& S(\omega)=\frac{\Delta V}{\sigma_{g}} \cdot \frac{\sigma_{g}}{\Delta p} \cdot \frac{\Delta p}{p_{1}}=S_{0}\left[\frac{1}{1-\left(\frac{\omega}{\omega_{0}}\right)^{2}}\right]\left[\frac{\Delta p}{p_{1}}\right],
\end{aligned}
$$

where:

$$
S_{0}=\left[\pi_{p z r} V_{b}\right]\left[\frac{S_{d}^{*}}{S_{g}}\right]\left[\frac{L / 2}{d}\right] \frac{d K_{g} d}{C_{h}+d K_{g} d},
$$

is the component that does not depend on the frequency.

Notice that the transfer function of the vibroacoustic problem $\left[\sigma_{g} / p_{1}\right]$ can be estimated in different ways depending on the model used. In the following, we present results obtained from the finite element model previously detailed and analytical results from a lumped model [24], [26].

The expression of the sensitivity indicates that the measured voltage fluctuation at the output of the bridge 29] is proportional to the difference in pressure for a pulsation lower than the resonant angular frequency of the mechanical structures. It also highlights the amplification 
provided by the mechanical architecture. The first amplification mechanism comes from the ratio between the modified lateral surface of a beam $S_{d}^{*}$ and section of a nanowire $S_{g}$ while the second comes from the ratio of the lever arm $(L / 2 d)$.

\subsubsection{Numerical results}

Figure 13 shows the microphone sensitivity curve for a configuration whose geometry is detailed in figure 8 . It shows the amplitude and phase transfer between the harmonic pressure imposed at the inlet and the voltage at the output of the strain gauge bridge. The sensitivity level of the sensor is substantially proportional to the incident pressure fluctuation. Its bandwidth is limited by the cutoff frequency $f_{1}$ conditioned by the viscous resistance of the micro-system, and the resonance frequency of the mechanical structures. Sensitivity at $1 \mathrm{kHz}$ is $5.6 \mathrm{mV} / \mathrm{Pa}$. The numerical and analytical lumped models [24], [26] give similar responses for the whole bandwidth. In these models the mechanical behavior of the beam is governed with the same analytical approach, however the acoustic models differ and is more precise in case of FEM. This difference is visible at the resonant frequency. It is mainly caused by a different way of estimating viscous resistance.

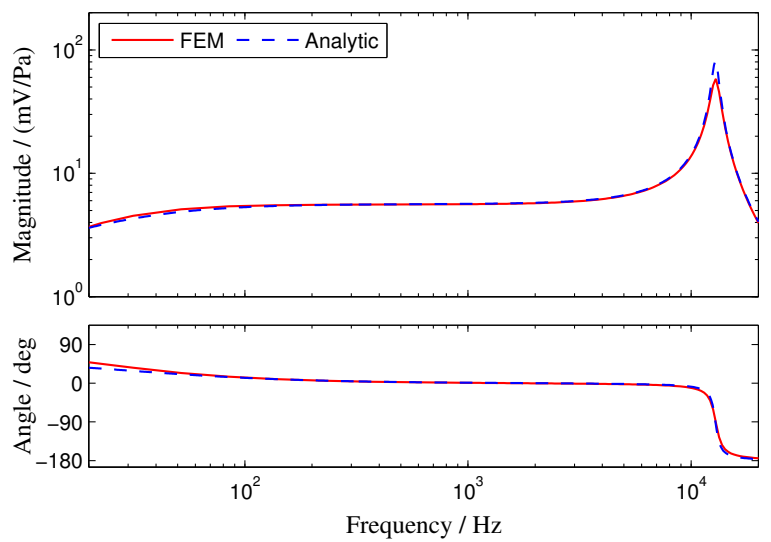

Figure 13. Pressure sensitivity $\Delta V / p_{1}$ (gap under the beams $1 \mu \mathrm{m}$, gap over the beams $2 \mu \mathrm{m}$ (see Figure 14) and back volume of $13 \mathrm{~mm}^{3}$ ).

\subsubsection{Influence of the thickness of the gap}

The thickness of the slit between the beams and the silicon layer of the SOI wafer is set to $1 \mu \mathrm{m}$ by the thickness of the initial oxide layer. However, the thickness of the slit between the beams and the cover can be adjusted between $1 \mu \mathrm{m}$ and $2 \mu \mathrm{m}$ during manufacturing (see figure 14). Indeed, the two oxide layers on the cover being removed for the release of the beam, one can reduce the thickness to a value close to $1 \mu \mathrm{m}$ by protecting one of the oxide layers with a metal deposit.

Figure 15 shows the influence of the thickness of the interstitial gap between the beams and the cover on the

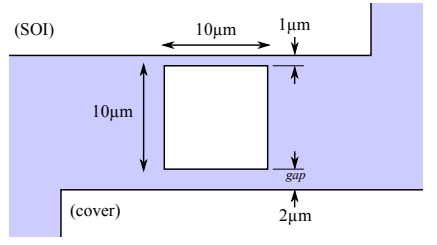

(a)

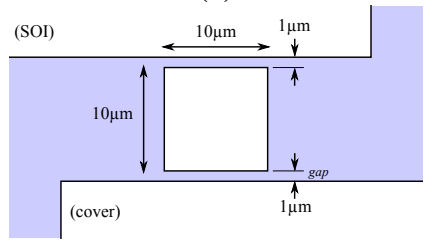

(b)

Figure 14. Geometries used to study the influence of the gap.

microphone sensitivity. The increase of the gap, which reduces the viscous resistance of the microphone, involves an increase of the cutoff frequency $f_{1}$ and a small loss damping of mechanical resonance. The viscous resistance of interstitial slots being preponderant, this slight variation of the gap has little influence on the microphone sensitivity in the passband.

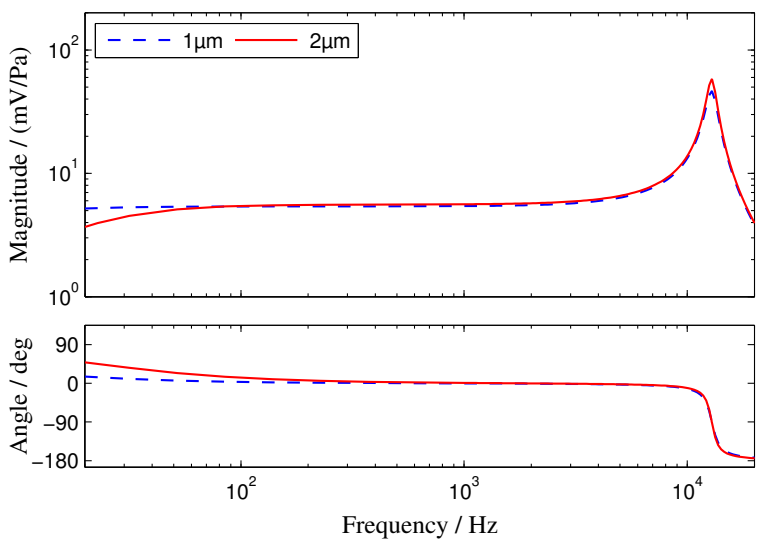

Figure 15 . Variation of pressure sensitivity with the gap values $1 \mu \mathrm{m}$ and $2 \mu \mathrm{m}$.

\subsubsection{Influence of the back volume.}

The package defines the volume of the back cavity and the size of the microphone. The miniaturization of the microphone means reducing the volume of the back cavity and therefore its acoustic flexibility. Figure 16 illustrates the influence of the rear volume on sensitivity, maintaining a gap of $2 \mu \mathrm{m}$. The cutoff frequency increases as the rear volume decreases. It reaches the critical value of $100 \mathrm{~Hz}$ for a volume of $3 \mathrm{~mm}^{3}$ (The back volume of $13 \mathrm{~mm}^{3}$ is the volume of the package that will be used to test the first prototypes). 


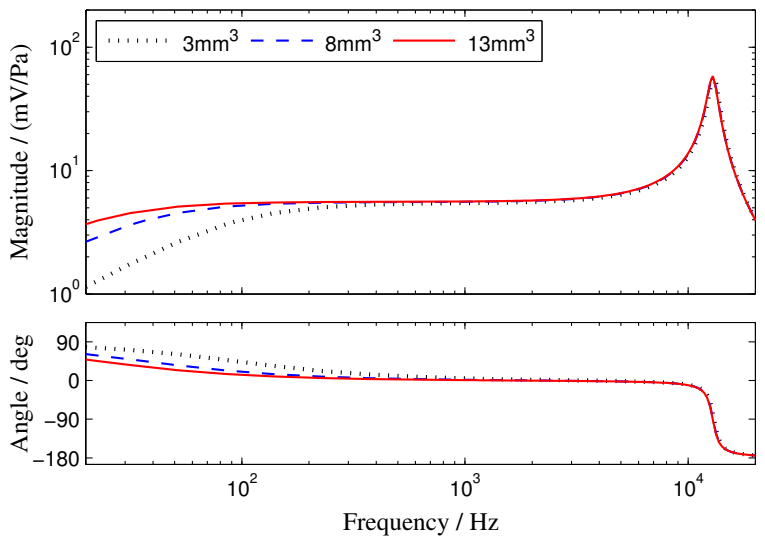

Figure 16. Variation of the pressure sensitivity with the back volume $\left(13 \mathrm{~mm}^{3}, 8 \mathrm{~mm}^{3}\right.$ and $\left.3 \mathrm{~mm}^{3}\right)$

\subsubsection{Influence of the geometry of the beam section}

2D modeling developed during our work considers a homogeneous and uniform beam section along the entire length of the micro-system. The viscous resistance of the couplers do not take into account the fact that the section of the truss changes with the cutting plane. To observe the influence of the geometry of the beams on the microphone sensitivity, we build a model in which the geometry of the beams corresponds to the section of a truss.

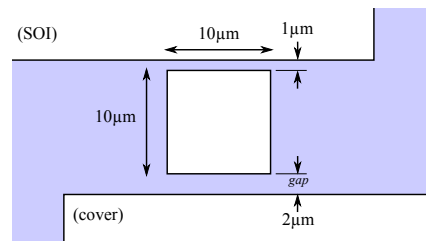

(a)

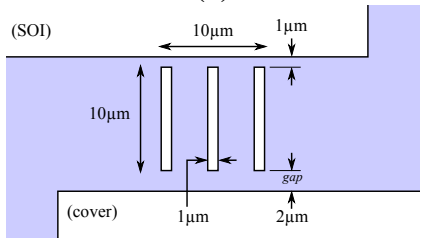

(b)

Figure 17. Geometry models used to study the influence of the section geometry. (a) solid section (b) truss section

Figure 19 compares the sensitivities obtained with a solid section and a hollow section (see Figure 17). The hollow section can be compared to the combination of three short slots arranged in series: for the hollow section, the reduction of the length of the interstitial slots induces a reduction of the viscous resistance in the coupler and a slight increase of the cut of frequency.

The change in section has little influence on the beam and the overall behavior is the same. Air located in the hollow section is driven by the beams with almost identical velocity.

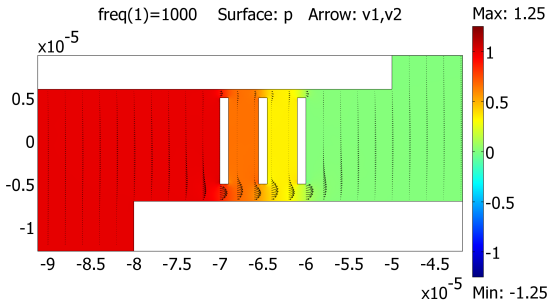

(a) $\quad f=1 \mathrm{kHz}$

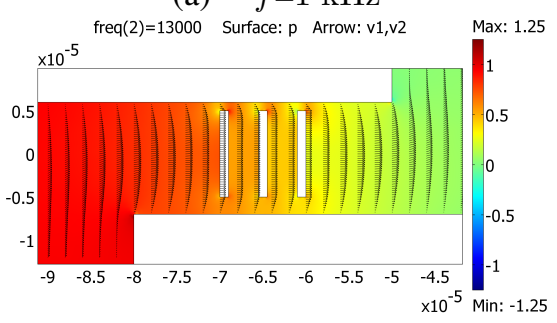

(b) $f=13 \mathrm{kHz}$

Figure 18. Pressure field (in color) and velocity profiles (arrows) in the coupler for a mobile beam of hollow section. Response to a unit harmonic pressure at $1 \mathrm{kHz}$ and $13 \mathrm{kHz}$ imposed on $S_{1}$ (Figure 5-b).
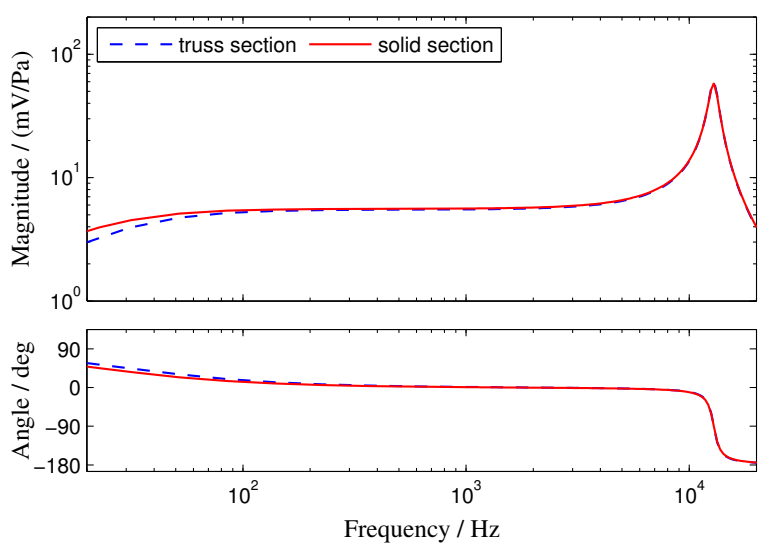

Figure 19. Sensitivity of the pressure obtained for a beam with a solid section (blue curve) and a truss section (red curve)

\section{Conclusion}

A new architecture of MEMS microphone based on micro-beams moving in the plane of the device and nanogauges detection was introduced. To design the MEMS microphone and assess its performances, a vibroacoustic model based on the Full Linearized Navier-Stokes equations (FLNS) was developed and discretized by the finite element method. The model allows a simulation of the acoustic behavior in a simplified equivalent planar microsystem. It simulates the acoustic behavior of the air coupled to micro-beams by imposing continuity of speeds on substructures interfaces as an auxiliary condition. The formulations used allow to solve problems in the time domain for the transitional regime and in the frequency domain for the determination of transfer functions of the microsystem. 
The results presented have shown the influence of the viscous effects on the acoustic behavior of the microsystem at low frequencies:

- when the micro-system is encapsulated, the viscous resistance of the micro-system and the back cavity determine the cutoff frequency. Above this frequency the pressure difference is sufficient for the operation of the micro-system,

- the shear viscosity which occurs at the flat slots (couplers) helps to maintain the pressure difference on both sides of the micro-structures. It ensures the separation between the front part subjected to pressure fluctuation and the rear part connected to the back cavity.

Sensitivity curves obtained by numerical simulation of the simplified micro-system are similar to those obtained from the analytical lumped model, demonstrating the efficiency of the models used. Influence of the geometrical parameters on the sensitivity was also studied. The volume of the back cavity appears as the most important parameter in the response curve.

Microfabrication process necessary to build such microphone architecture is carried out at CEA-LETI. The developed models in this article have provided a better understanding of the physical mechanisms involved and the important parameters of this new sensor.The experimental characterization of first prototypes will be necessary to validate the models presented in this article.

\section{Acknowledgement}

This work was performed within the framework of the Labex CeLyA of Universite de Lyon, operated by the French National Research Agency (ANR-10-LABX-0060/ ANR-11-IDEX-0007). Development of M\&NEMS microphone was supported by the French National Research Agency (MADNEMS project ANR11-NANO-026).

\section{References}

[1] E. Wente: A condenser transmitter as a uniformly sensitive instrument for the absolute measurement of sound intensity. Physical Review 10(1) (1917) 39-63.

[2] G. Sessler, J. West: Self-biased condenser microphone with high capacitance. The Journal of the Acoustical Society of America 34 (1962) 1787-1788.

[3] D. Hohm, G. M. Sessler: An integrated silicon-electretcondenser microphone. Proc. of the 11 th Int. Congress on Accoustics, Paris 6 (1983) 29-32.

[4] K. E. Petersen: Silicon as a mechanical material. Proceedings of the IEEE 70(5) (1982) 420-457.

[5] P. Robert, P. Rey, A. Berthelot, G. Jourdan, Y. Deimerly, S. Louwers, J. Bon, F. Boillot, J. Collet: M\&nems: a technological platform for 10-axis sensor. SSI Conference, Amsterdam, (2013).

[6] W. Kronast, B. Muller, W. Siedel, A. Stoffel: Single-chip condenser microphone using porous silicon as sacrificial layer for the air gap. Sensors and Actuators A: Physical 87(3) (2001) 188-193.
[7] M. Kuntzman, C. Garcia, A. Onaran, B. Avenson, K. Kirk, N. Hall: Performance and modeling of a fully packaged micromachined optical microphone. Journal of Microelectromechanical Systems 20(4) (2011) 828-833.

[8] J. Weigold, T. Brosnihan, J. Bergeron, X. Zhang: A mems condenser microphone for consumer applications. 19th IEEE International Conference on Micro Electro Mechanical Systems, Istanbul (2006) 86-89.

[9] C. Chun-Kai, L. Wei-Cheng, W. Mingching, W. MingYung, F. Weileun: Design and implementation of a capacitive-type microphone with rigid diaphragm and flexible spring using the two poly silicon micro-machining processes. Sensors Journal, IEEE 11(10) (2011) 2365-2371.

[10] Y. Iguchi, M. Goto, M. Iwaki, A. Ando, K. Tanioka, T. Tajima, F. Takeshi, S. Matsunaga, Y. Yasuno: Silicon microphone with wide frequency range and high linearity. Sensors and Actuators A: Physical 135(2) (2007) 420-425.

[11] A. Dehe, M. Wurzer, M. Fuldner, U. Krumbein: The infineon silicon mems microphone. Proceedings SENSOR 2013 (2013) 95-96.

[12] A. Walther, M. Savoye, G. Jourdan, P. Renaux, F. Souchon, P. Robert, C. L. Blanc, N. Delorme, O. Gigan, C. Lejuste: 3 axis gyroscope with si nanogage piezo-resistive detection. Proc. IEEE MEMS (2012).

[13] M. Bruneau, T. Scelo (translator and contributor): Fundamentals of acoustics. ISTE, London, 2006.

[14] T. L. V. Suu, S. Durand, M. Bruneau: On the modelling of clamped plates loaded by a squeeze fluid film: application to miniaturised sensors. Acta Acust. united Ac. 96 (2010) 923-935.

[15] T. Lavergne, S. Durand, M. Bruneau, N. Joly: Dynamic behavior of the circular membrane of an electrostatic microphone: Effect of holes in the backin electrode. J. Acoust. Soc. Am. 128 (2010) 3459-3477.

[16] C. Karra, M. B. Tahar: Boundary element analysis of vibratory behaviour of a membrane coupled to a thermal conductor layer fluid. C. R. Académie des Sciences, Série II B - Mécanique Physique Astronomie 326 (11) (1998) 733 739 .

[17] V. C. Henriquez, P. Juhl: An axisymmetric boundary element formulation of sound wave propagation in fluids including viscous and thermal losses. J. Acoust. Soc. Am. 134 (5) (2013) 3409-3418.

[18] V. C. Henriquez, P. Juhl: Implementation of an acoustic 3d bem with visco-thermal losses. Proc. Internoise 2013, Innsbruck (Austria), (2013).

[19] R. Bossart, N. Joly, M. Bruneau: Methods for the numerical modeling of acoustic fields in a thermoviscous fluid. Proc. of the 6th French Congress of Acoustics, Lille (France), (2002), 411-414.

[20] M. Malinen, M. Lyly, P. Råback, A. Kärkkäinen, L. Kärkkäinen: A finite element method for the modeling of thermo-viscous effects in acoustics. Proc. of the 4th European Congress Computational Methods in Applied Sciences and Engineering ECCOMAS, Jyväskylä (Finland), (2004), 1-12.

[21] H. I. Hussain, J.-L. Guyader: Sound radiation of plates in thermoviscous media. Acta Acust. united Ac 95 (6) (2009) 997-1005.

[22] N. Joly: Finite element modeling of thermoviscous acoustics on adapted anisotropic meshes: Implementation of the particle velocity and temperature variation formulation. Acta Acust. united Ac. 96 (2) (2010) 102-114. 
[23] W. R. Kampinga, Y. H. Wijnant, A. de Boer: An efficient finite element model for viscothermal acoustics. Acta Acust. united Ac. 97 (4) (2011) 618-631.

[24] C. Guianvarc'h, T. Verdot, J. Czarny, E. Redon, K. Ege, J.L. Guyader, A. Walther, P. Robert: New planar nano-gauge detection microphone: analytical and numerical acoustic modelling. Proc. International Congress on Acoustics, Montreal, (2013), 1-9.

[25] T. Verdot, C. Guianvarc'h, J. Czarny, E. Redon, K. Ege, J.-L. Guyader: Modélisation d'une architecture innovante de microphone mems à détection par nano jauges. Proc. Congrès Français de Mécanique, Bordeaux, (2013), 1-6.

[26] C. Guianvarc'h: La cavité de couplage acoustique dans la méthode de réciprocité : modèles analytiques pour l'étalonnage des microphones et la mesure d'impédances de petits composants. PhD thesis, Université du Maine, 2005. 